\title{
A SMAP in the face for cancer
}

\author{
Shirish Shenolikar \\ Program in Cardiovascular and Metabolic Disorders, Duke-NUS Medical School Singapore, Singapore.
}

\begin{abstract}
Observed deficits in protein phosphatase 2A (PP2A) function in a variety of human cancers have stimulated drug discovery efforts aimed at restoring PP2A function to inhibit tumor growth. Work published by Sangodkar et al. in this issue of the $J C I$ describes the characterization of orally available small molecule activators of PP2A (SMAPs). These SMAPs attenuated mitogenic signaling and triggered apoptosis in KRAS-mutant lung cancer cells and inhibited tumor growth in murine models. Tumors with mutations in the SMAP-binding site of the PP2A A subunit displayed resistance to SMAPs. Future studies that identify the PP2A-regulated events targeted by SMAPs should guide critical decisions about which cancers might be best treated with these molecules. This study provides encouraging evidence in favor of SMAPs as potential anticancer drugs.
\end{abstract}

phosphatases are inhibited by these compounds and to what level phosphatase activity must be reduced to promote cell proliferation versus cell death.

Evidence pointing to a specific role for PP2A in human tumorigenesis came from the observation that small $\mathrm{T}$ antigen of simian virus 40 (SV40) and middle $\mathrm{T}$ antigen of polyomavirus bind PP2A heterodimers (composed of $\mathrm{A}$ and $\mathrm{B}$ subunits), potentially displacing or precluding the recruitment of $\mathrm{B}$ subunits that define the subcellular localization and substrate specificity of cellular PP2A heterotrimers (composed of $\mathrm{A}, \mathrm{B}$, and $\mathrm{C}$ subunits). By perturbing these complexes, SV40 small T antigen inhibits PP2A function, which is now recognized as a key requirement for transforming human cells (5). Other studies showed that the loss of B56 $\gamma$ function substitutes for the expression of SV 40 small $\mathrm{T}$ antigen in the transformation assay (6). Together, these data indicate that negating the function of some but not all PP2A heterotrimers facilitates cell transformation. Curiously, polyomavirus and SV40 antigens target distinct as well as common PP2A heterotrimers (7) and modulate different PP2A-regulated pathways to enhance cell growth (8), suggesting that there may be multiple PP2A-regulated pathways involved in oncogenesis.

Further evidence favoring PP2A as a tumor suppressor came from the identification of numerous mutations in PP2A subunits in human cancers, with the preponderance of these mutations being in the A subunit. Two mammalian genes encode PP2A A subunits, and cancer-associated mutations have been identified in both the $A \alpha$ and $A \beta$ isoforms. Interestingly, the abundance of $A \alpha$ far exceeds that of $A \beta$ in most cells, and despite their high homology, tumorigenesis elicited by a reduction in $A \beta$ function is not suppressed by increasing $A \alpha$ levels. These and other data (9) strongly suggest that these A subunits assemble functionally distinct PP2A complexes, which in turn play unique roles in tumor development. 


\section{Strategies for activating PP2A}

Given that various estimates point to more than 250,000 potential phosphorylation sites, the vast majority of which are on serine and threonine residues, in mammalian cells and that PP2A accounts for more than $50 \%$ of cellular protein serine/threonine phosphatase activity, it should come as no surprise that PP2A is tightly controlled by a number of different mechanisms. These different modes of regulation include the assembly of 80 or more PP2A complexes, the presence of both PP2A inhibitors and activators, and covalent modifications of PP2A subunits. Some of these regulatory mechanisms form the bases for drug design strategies that are currently being pursued to reactivate $\mathrm{PP} 2 \mathrm{~A}$ and restore tumor suppressor activity in human tumors (10).

One such example is $\mathrm{I}_{2} \mathrm{PP} 2 \mathrm{~A}$, also known as SET, an endogenous protein inhibitor of PP2A. SET is overexpressed in human cancers, including chronic myeloid leukemia as well as colorectal, breast, and lung cancers (11). Translocation of the human gene encoding SET has also been linked to leukemia. SET function can be counteracted by the sphingolipid ceramide, which activates $\mathrm{PP} 2 \mathrm{~A}$, at least in part by disrupting the association of SET with PP2A to trigger apoptosis. FTY720, a drug currently approved for the treatment of multiple sclerosis, also impairs the PP2ASET interaction to activate PP2A. Moreover, FTY720 shows antitumor activity in several human cancer models and may be particularly effective in eradicating myeloid leukemia (12). Unlike ceramide, which also activates PP1, FTY720 does not appear to target phosphatases other than PP2A and thus represents a good starting point for the future development of anticancer therapies.

Another strategy exploits the methylation of PP2A catalytic subunit (C subunit) near the $C$ terminus (at Leucine-309). This modification is catalyzed by the methyltransferase LCMT-1 and reversed by the methyl esterase PME-1, the latter being remarkably selective for the PP2A C subunit. As structural and functional data have argued that $\mathrm{C}$ subunit methylation plays an important role in the assembly of the PP2A heterotrimer, small molecule inhibiters of PME-1 have been developed with the anticipation that these molecules will increase cellular PP2A content or activity. However, methylation at Leucine-309 appears to be critical for assembly of some PP2A heterotrimers and not others. In this regard, PP2A heterotrimers containing the B55a subunit, which is predominantly expressed in brain, appear to be particularly reliant on $\mathrm{C}$ subunit methylation. As increased PME-1 activity has been implicated in the resistance of gliomas to a variety of kinase inhibitors (13), one could speculate that PME-1 inhibitors might be particularly useful in the treatment of gliomas and other brain disorders, such as Alzheimer's and Parkinson's disease, that have been linked to reduced PP2A function.

\section{SMAPs: new kids in town}

In a screen of FDA-approved drugs, Gutierrez and colleagues (14) first noted that a subset of phenothiazines induced apoptosis in lymphoblastic leukemia cells. Ligand affinity chromatography identified PP2A as a potential perphenazine target, and the drug-induced changes in phosphoproteins, in vitro and in vivo, pointed to PP2A activation as the likely mechanism underlying the anticancer activity of these drugs. Subsequent reengineering of these compounds eliminated their CNS effects while retaining and/or even enhancing their anticancer activity. In this issue, Sangodkar et al. (15) now show that these reengineered compounds, termed small molecule activators of PP2A (SMAPs), share the ability to activate PP2A. Biochemical studies identified the PP2A A subunit as the primary target of SMAPs, specifically the centrally located HEAT repeats five to eight. In tumors, mutation of the proposed SMAP-binding sequence in the A subunit conferred resistance to SMAPs, establishing PP2A as the primary cellular target of SMAPs responsible for tumor suppression. Despite the overlap in the SMAP-binding site with that of B subunits in the A subunit, SMAPs were shown to differ from other PP2A activators in that they activated the PP2A (AC) heterodimer and the PP2A (AB56C) heterotrimer in a nearly identical manner, suggesting that the presence of the B subunit (at least B56) does not hinder drug binding. Moreover, mutations in the A subunit that attenuated SMAP binding did not influence assembly of the PP2A ABC heterotrimer. While not conclusive, these data may point to SMAPs as a new class of PP2A activators that dif- fer from other PP2A activators in targeting a broader array of PP2A complexes. The ability to target multiple PP2A complexes in turn should raise some concerns as to whether SMAPs might, while treating cancer, trigger some other disease. However, comparison of the SMAP dose-response curves for PP2A activation and tumor suppression highlights that only modest PP2A activation was needed for effective inhibition of tumor growth. This property may narrow the PP2A populations affected and thereby curtail any potential for SMAPs to cause serious harm.

\section{The road ahead...}

Cancer places an enormous burden on society, driving the recent efforts by academic and industry scientists to identify novel anticancer drugs. The recognition that PP2A function is reduced in many cancers has prompted a multipronged search for PP2A activators that display antitumor activity. SMAPs represent the latest in this rapidly growing line of $\mathrm{PP} 2 \mathrm{~A}$-directed therapeutics that show promise in impeding tumor growth. The above discussion also highlights the gaps in knowledge of the biochemistry and cell biology of PP2A in cancer cells. Thus, our inability to predict whether expression levels or subcellular localization of PP2A complexes might influence drug efficacy and the unknown identity of the PP2A substrates in cancer and noncancer cells that can function as biomarkers for drug administration and efficacy or inform on potential side effects may become serious hurdles in taking SMAPs and other PP2A activators into the clinic. The lack of knowledge about the signaling pathways modulated by SMAPs will also hinder the identification of cancers that would be most effectively treated with SMAPs. These hurdles must be addressed, sooner rather than later, if SMAPs and other PP2A-targeted therapies are to become a reality as treatments for human cancers.

\section{Acknowledgments}

Work in our lab is supported by a National Parkinson's Disease Translational Clinical Research Programme supported by the National Medical Research Council (NMRC/TCR/013-NNI/2014) and additional funding provided through the DukeNUS Medical School by the Ministry of Health, Singapore. 
Address correspondence to: Shirish Shenolikar, Program in Cardiovascular and Metabolic Disorders, Duke-NUS Medical School Singapore, 8 College Road, Singapore 169857, Singapore. Phone: 65.6221.8625; E-mail: shirish.shenolikar@ duke-NUS.edu.sg.

1. Gross S, Rahal R, Stransky N, Lengauer C, Hoeflich KP. Targeting cancer with kinase inhibitors. J Clin Invest. 2015;125(5):1780-1789.

2. Bialojan C, Takai A. Inhibitory effect of a marine-sponge toxin, okadaic acid, on protein phosphatases. Specificity and kinetics. Biochem J. 1988;256(1):283-290.

3. Honkanen RE, Codispoti BA, Tse K, Boynton AL, Honkanan RE. Characterization of natural toxins with inhibitory activity against serine/ threonine protein phosphatases. Toxicon. $1994 ; 32(3): 339-350$
4. Scheithauer W, Von Hoff DD, Clark GM, Shillis JL, Elslager EF. In vitro activity of the novel antitumor antibiotic fostriecin (CI-920) in a human tumor cloning assay. Eur J Cancer Clin Oncol. 1986;22(8):921-926.

5. Sablina AA, et al. The tumor suppressor PP2A A $\beta$ regulates the RalA GTPase. Cell. 2007;129(5):969-982.

6. Chen W, Possemato R, Campbell KT, Plattner CA, Pallas DC, Hahn WC. Identification of specific PP2A complexes involved in human cell transformation. Cancer Cell. 2004;5(2):127-136.

7. Kwun HJ, et al. Restricted protein phosphatase 2A targeting by Merkel cell polyomavirus small T antigen. J Virol. 2015;89(8):4191-4200.

8. Powsner ER. Comments on the flow cytometry analysis program of J. Fried and M. Mandel. Comput Programs Biomed. 1981;13(3-4):291-292.

9. Sablina AA, Hahn WC. SV40 small T antigen and PP2A phosphatase in cell transformation. Cancer Metastasis Rev. 2008;27(2):137-146.

10. Ruvolo PP. The broken "Off" switch in cancer signaling: PP2A as a regulator of tumorigenesis, drug resistance, and immune surveillance. $B B A$ Clin. 2016;6:87-99.

11. Sangodkar J, Farrington CC, McClinch K, Galsky MD, Kastrinsky DB, Narla G. All roads lead to PP2A: exploiting the therapeutic potential of this phosphatase. FEBS J. 2016;283(6):1004-1024.

12. Neviani $P$, et al. PP2A-activating drugs selectively eradicate TKI-resistant chronic myeloid leukemic stem cells. J Clin Invest. 2013;123(10):4144-4157.

13. Kaur A, et al. PP2A Inhibitor PME-1 drives kinase inhibitor resistance in glioma cells. Cancer Res. 2016;76(23):7001-7011.

14. Gutierrez A, et al. Phenothiazines induce PP2A-mediated apoptosis in T cell acute lymphoblastic leukemia. J Clin Invest. 2014;124(2):644-655.

15. Sangodkar J, et al. Activation of tumor suppressor protein PP2A inhibits KRAS-driven tumor growth. JClin Invest. 2017;127(6):2081-2090. 